\title{
Delay Analysis on-chip VLSI Interconnect using Gamma Distribution Function
}

\author{
R. Kar \\ VLSI Laboratory \\ Department of ECE \\ NIT Durgapur-713209
}

\author{
V. Maheshwari \\ VLSI Laboratory \\ Department of ECE \\ NIT Durgapur-713209
}

\author{
Ashis K. mal \\ VLSI Laboratory \\ Department of ECE \\ NIT Durgapur-713209
}

\author{
A.K.Bhattacharjee \\ VLSI Laboratory \\ Department of ECE \\ NIT Durgapur-713209
}

\begin{abstract}
Moments of the impulse response are widely used for interconnect delay analysis, from the explicit Elmore delay (the first moment of the impulse response) expression, to moment matching methods which creates reduced order trans-impedance and transfer function approximations. However, the Elmore delay is fast becoming ineffective for deep submicron technologies, and reduced order transfer function delays are impractical for use as early-phase design metrics or as design optimization cost functions. This paper describes an approach for fitting moments of the impulse response to probability density functions so that delay can be estimated accurately at an early physical design stage. For RC trees it is demonstrated that the incomplete gamma function provides a provably stable approximation. The accuracy of our model is justified with the results compared with that of SPICE simulations.
\end{abstract}

\section{Categories and Subject Descriptors}

B.7.2 [Integrated Circuits]: Design Aids - Simulation;

\section{General Terms}

Algorithms, Design, Theory

\section{Keywords}

Moment Matching, On-Chip Interconnect, Probability Distribution Function, Delay calculation, Gamma Distribution, VLSI.

\section{INTRODUCTION}

The advent of sub-quarter-micron IC technologies has forced dramatic changes in the design and manufacturing methodologies for integrated circuits and systems. The paradigm shift for interconnect which was once considered just a parasitic but can now be the dominant factor to determine the integrated circuit performances. It results the greatest impetus for change of existing methodologies. Over the past decade there have been a number of advances in modeling and analysis of interconnect that have facilitated the continual advances in design automation for systems of increasing frequency and downsizing. As integrated circuit feature sizes continue to scale well below 0.18 microns, active device counts are reaching hundreds of millions [3]. The amount of interconnect among the devices tends to grow super linearly with the transistor counts, and the chip area is often limited by the physical interconnect area. Due to these interconnect area limitations, the interconnect dimensions are scaled with the devices whenever possible. In addition, to provide more wiring resources, IC's now accommodate numerous metallization layers, with more to come in the future. These advances in technology that result in scaled, multi-level interconnects may address the wire-ability problem, but in the process creates problems with signal integrity and interconnect delay. This paper proposes an extension of Elmore's approximation [1] to include matching of higher order moments of the probability density function. Specifically, using a timeshifted incomplete Gamma function approximation [2] for the impulse responses of $\mathrm{RC}$ trees, the three parameters of this model are fitted by matching the first three central moments (mean, variance, skewness), which is equivalent to matching the first two moments of the circuit response (m1, m2,). Importantly, it is proven that such a gamma fit is guaranteed to be realizable and stable for the moments of an RC tree [4]. Once the moments are fitted to characterize the Gamma function, the step response delay is obtained as a closed form expression thereby providing the same explicitness as the Elmore approximation. This work is simple yet accurate compared to the model proposed in [4] in respect that our approach provides a closed form expression and does not require any look up table to calculate the delay for RC interconnect as required in [4].

\section{BASIC THEORY}

\subsection{Moments of a Linear Circuit Response}

Let $\mathrm{h}(\mathrm{t})$ be a circuit impulse response in the time domain and let $\mathrm{H}(\mathrm{s})$ be the corresponding transfer function. By definition, $\mathrm{H}(\mathrm{s})$ is the Laplace transform of $\mathrm{h}(\mathrm{t})$ [12],

$H(s)=\int_{0}^{\pi} h(t) e^{-s t} d t$

Applying a Taylor series expansion of $e^{-s t}$ about $\mathrm{s}=0$ yields,

$H(s)-\sum_{t=0} \frac{(-1)}{i !} s^{t} \int_{0}^{m} t^{t} h(t) d t$

The $i^{t h}$ circuit-response moment, $\tilde{m}_{i}$ is defined as [5]:

$$
\tilde{m}_{i}=\frac{(-1)^{i}}{i !} \int_{0}^{\infty} t^{i} h(t) d t
$$


From (2) and (3), the transfer function $H$ (s) can be expressed as:

$$
H(s)=\tilde{m}_{0}+\tilde{m}_{1} s+\tilde{m}_{3} s^{3}+\tilde{m}_{1} s^{\mathbf{l}}+\ldots
$$

\subsection{Central Moments}

It is straightforward to show that the first few central moments can be expressed in terms of circuit moments as follows [6]:

$$
\left.\begin{array}{l}
\mu_{0}=m_{0}, \mu_{1}=0, \mu_{2}=2 m_{2}-\frac{m_{1}^{2}}{m_{0}}, \\
\mu_{3}=-6 m_{3}+6 \frac{m_{1} m_{2}}{m_{0}}-2 \frac{m_{1}^{3}}{m_{0}^{2}}
\end{array}\right\}
$$

Unlike the moments of the impulse response, the central moments have geometrical interpretations:

$\mu_{0}$ is the area under the curve. It is generally unity, or else a simple scaling factor is applied.

$\mu_{2}$ is the variance of the distribution which measures the spread or the dispersion of the curve from the center. A larger variance reflects a larger spread of the curve.

$\mu_{3}$ is a measure of the skewness of the distribution; for a unimodel function, its sign determines whether the mode (global maximum) is to the left or to the right of the expected value (mean). Its magnitude is a measure of the distance between the mode and the mean.

\subsection{Higher Central Moments in RC Tress}

The second and third central moments are always positive for RC tree impulse responses [6]. The positiveness of the second order central moment is obvious from its definition

$$
\mu_{2}=\int_{0}^{\infty}(t-\mu)^{2} h(t) d t
$$

The impulse response, $\mathrm{h}(\mathrm{t})$, at any node in an $\mathrm{RC}$ tree is always positive. Hence the second central moment $\mu_{2}$ is always positive.

\section{PROPOSED MODEL}

Elmore's original delay approximation is based on the analogy between non-negative impulse responses and probability density functions. In theory, Elmore's distribution interpretation can be extended beyond simply estimating the median by the mean if higher order moments can be used to characterize a representative distribution function. Once characterized, the delay can be approximated via closed form expression or table lookup of the median value for the representative distribution family. One proposal was to use a gamma distribution function [4]. Although [4] is not an explicit one. The gamma distribution is a reasonably good representation of $\mathrm{RC}$ tree impulse responses since it provides good "coverage" of bell shaped curves which are bounded on the left and exponentially decaying to the right [10]. The Gamma distribution is depicted in Figure (1) [11]. The probability density function of gamma distribution $g_{\lambda, n}(t)$, is a function of one variable $t$ and two parameters $\lambda$ and $n$ (positive real numbers) [11]

$$
g_{\lambda, n}(t)=\frac{\lambda^{n} t^{n-1} e^{-\lambda t}}{\Gamma(n)}, t>0
$$

Where, $\Gamma(\mathrm{x})$ is the gamma function defined as:

$$
\Gamma(x)=\int_{0}^{\infty} y^{x-1} e^{-y} d y, x>0
$$

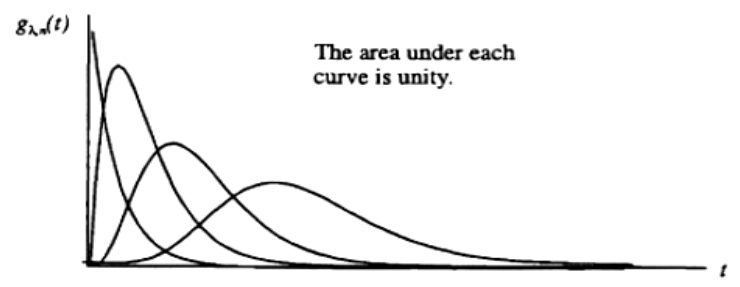

Figure 1. The Gamma Distribution Function

Now consider an impulse response $\mathrm{h}(\mathrm{t})$ and assume that it is approximated with a gamma probability density function

$h(t)=g_{\lambda, n}(t)$

Then the transfer function is given by:

$H(s)=\left(\frac{\lambda}{\lambda+s}\right)^{n}$

The s-domain model denoted by (10) may be interpreted as a unique pole with a real number order. Notice that when $n=1$, the gamma distribution model naturally degrades to the dominant pole model. However, the existence of the parameter $\mathrm{n}$ increases the degree of freedom of the model. The first few moments of the transfer function can be expressed easily in terms of the parameters $\mathrm{n}$ and $\lambda[2]$.

$m_{0}=1, m_{1}=-\frac{n}{\lambda}, m_{2}=\frac{n(n+1)}{2 \lambda^{2}}, m_{3}=\frac{n(n+1)(n+2)}{6 \lambda^{2}}$

Since the gamma function has only two variables, it can be uniquely characterized by fitting it with two moments [2], [11]. For example, using the second and third equations, the parameters $\lambda$ and $\mathrm{n}$ can be obtained from the first and second order circuit moments as:

$\lambda=\frac{m_{1}}{m_{1}^{2}-2 m_{2}}, n=\frac{-m_{1}^{2}}{m_{1}^{2}-2 m_{2}}$

In terms of the second central moment becomes

$\lambda=\frac{-m_{1}}{\mu_{2}}$ and $\quad n=\frac{m_{1}^{2}}{\mu_{2}}$

Recall that both $-\mathrm{m}_{1}$ the Elmore delay) and $\mu_{2}$ are positive numbers for RC trees. Therefore the impulse response approximation with the parameters is always stable. 
In the approximation above, we have used the first two moments. However, at least three moments are generally required to capture essential waveform response characteristics. Therefore, to match the third moment and capture the skewness of the distribution, we add a third variable $\Delta$, to include one more degree of freedom. We shift the gamma function $\Delta$ by to approximate the impulse response:

$$
h(t)=g_{1, n}(t-\Delta)
$$

Thus the transfer function becomes

$$
H(s)=e^{-s \Delta}\left(\frac{\lambda}{\lambda+s}\right)^{n}
$$

And, its moments are given by,

$$
\left.\begin{array}{l}
m_{1}=-\frac{n}{\lambda}-\Delta \\
m_{2}=\frac{n(n+1)}{2 \lambda^{2}}+\frac{n}{\lambda} \Delta+\frac{\Delta^{2}}{2} \\
m_{3}=-\frac{n(n+1)(n+2)}{6 \lambda^{3}}-\frac{n(n+1)}{2 \lambda^{2}} \Delta-\frac{n}{\lambda} \frac{\Delta^{2}}{2}-\frac{\Delta^{3}}{6}
\end{array}\right\}
$$

With $\mathrm{m}_{0}=1$. We now have three unknowns, $\lambda, \mathrm{n}$ and $\Delta$ in three equations. It can be shown that this equation system can also be expressed in terms of $\mathrm{m}_{1}$ and second and third central moments, $\mu_{2}$ and $\mu_{3}$.

$$
\left.\begin{array}{l}
m_{1}=-\frac{n}{\lambda}-\Delta \\
\mu_{2}=-\frac{n(n+1)}{\lambda^{2}}-\left(\frac{n}{\lambda}\right)^{2}=\frac{n}{\lambda^{2}} \\
\mu_{3}=\frac{n(n+1)(n+2)}{\lambda^{3}}-3\left(\frac{n}{\lambda}\right) \frac{n(n+1)}{\lambda^{2}}+2\left(\frac{n}{\lambda}\right)^{3}=\frac{2 n}{\lambda^{3}}
\end{array}\right\}
$$

Note that the second and third order central moments $\mu_{2}$ and $\mu_{3}$ are independent of the shift $\Delta . \lambda$ and $n$ are the measures of variance and skewness of the waveform, respectively, and they are not affected by the time shift.

This result,

$\lambda=\frac{2 \mu_{2}}{\mu_{3}}, n=\frac{4 \mu_{2}^{3}}{\mu_{3}^{2}}, \Delta=-m_{1}-\frac{n}{\lambda}$

We now find an expression for the step response, $y(t)$, which is the integral of the impulse response,

$$
y(t)-\int_{0}^{r} h(r) b r
$$

After some algebraic manipulation we obtain,

$y(t)=\int_{0}^{z, t-\Delta)} g_{3, n}(\tau) d \tau=P(n, \not \partial(t-\Delta))$

Where $\mathrm{P}(\mathrm{n}, \mathrm{x})$ is the incomplete gamma function [7-8].

$$
P(n, \lambda)=\frac{1}{\Gamma(n)} \int_{0}^{t} x^{n-1} e^{-x} d x
$$

Where $\Gamma(\mathrm{n})$ is the gamma function. Note that $\mathrm{P}(\mathrm{n}, \mathrm{t})$ is zero at $\mathrm{t}$ $=0$ and monotonically increases to one. Hence, to calculate the delay at a particular percentage point we only need to find the value of $\mathrm{x}$ such that

$P(n, x)=\alpha$

Where $\mathrm{x}=\lambda(\mathrm{t}-\Delta)$. Then with a simple scaling and shifting, the delay is obtained:

$t_{\alpha}=\frac{x}{\lambda}+\Delta$

\subsection{Calculation of Median of the Gamma Distribution Function}

Since gamma has only two parameters $\lambda$ and $\mathrm{n}$ matching two moments would completely characterize this model. Hence Mean of the Gamma function is given by

$$
\frac{n}{\lambda}=-m_{1}
$$

Variance of the Gamma function is given by

$$
\frac{n}{\hbar^{2}}=-\left(m_{\mathrm{L}}^{2}-2 m_{2}\right)
$$

From (23) and (24),

$$
\lambda=\frac{m_{1}}{m_{1}^{2}-2 m_{2}} \text { and } n=\frac{-m_{1}^{2}}{m_{1}^{2}-2 m_{2}}
$$

Now Mode of the Gamma function is given by $\frac{n-1}{\lambda}$.

Median of Gamma function is given by Mode $=3 *$ Median $-2 *$ Mean

$$
\left.\begin{array}{rl}
\text { Median } & =\frac{\text { Mode }+2 \text { Mean }}{3}=\left[\frac{(n-1)}{\lambda}+2 \frac{n}{\lambda}\right] / 3 \\
& =\left[3 \frac{n}{\lambda}-\frac{1}{\lambda}\right] / 3=\left[\frac{n}{\lambda}-\frac{1}{3 \lambda}\right]
\end{array}\right\}
$$

From (25) and (26),

$$
\left.\begin{array}{l}
\text { Median }=-m_{1}-\left(\frac{m_{1}^{2}-2 m_{2}}{3 m_{1}}\right) \\
\text { or, Delay }(50 \%)=-\frac{4}{3} m_{1}+\frac{2}{3} \frac{m_{2}}{m_{1}}
\end{array}\right\}
$$

From the above derived Formula for the Median of Gamma function we see that the Delay function is a simple function of the two first circuit moments. This is our proposed closed form delay model.

\section{EXPERIMENTAL RESULTS}

We have implemented the proposed delay estimation method using Gamma Distribution and applied it to widely used actual interconnect RC networks as shown in Figure 2. For each RC network source we put a driver, where the driver is a voltage source followed by a resister. 


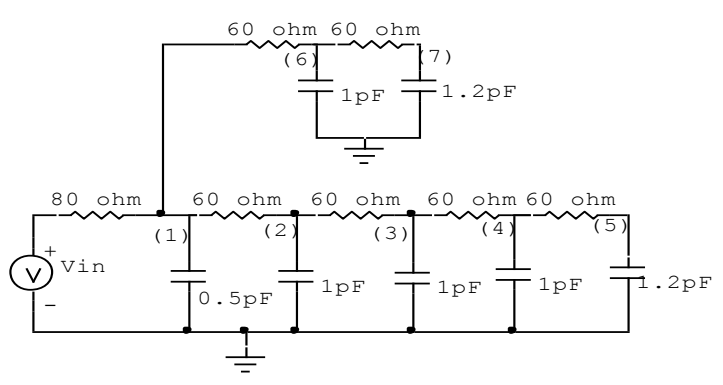

Figure 2. An RC Tree Example

Note that the difference between the Gamma Delay and the SPICE delay at the leaf nodes is about $1 \%$. The worst case delay obtained for the nodes that are closest to the node 2 . These nodes correspond to the responses with the highest frequency element,

Table 1. Comparison of the $50 \%$ delays between SPICE and the Proposed Delay Metric (time in ns).

\begin{tabular}{|c|c|c|}
\hline Node & $\begin{array}{c}\text { SPICE } \\
(\mathbf{n s})\end{array}$ & $\begin{array}{c}\text { Proposed Model } \\
(\mathbf{n s})\end{array}$ \\
\hline 1 & 0.196 & 0.234 \\
\hline 2 & 0.477 & 0.493 \\
\hline 3 & 0.700 & 0.697 \\
\hline 4 & 0.845 & 0.828 \\
\hline 5 & 0.919 & 0.923 \\
\hline 6 & 0.375 & 0.373 \\
\hline 7 & 0.452 & 0.451 \\
\hline
\end{tabular}

therefore one would expect the largest moment matching error.

This technique requires a bell-shaped distribution for the impulse response, over damped and critically damped RC circuit responses. The impulse response at the driving point node does not follow the bell shape, but rather starts out with a non-zero value and asymptotically approach zero in a multi-exponential decay form. Other distribution families may permit matching higher order moments, or more naturally capture these driving point response shapes. But as with all moment matching problems, the greatest challenge is to find a model that is provably stable and realizable.

\section{CONCLUSION}

In this paper we have proposed an efficient and accurate interconnect delay metric for high speed VLSI designs. We have used Gamma probability distribution function to derive our metric. Our model has Elmore delay as upper bound but with significantly less error. The novelty of our approach is justified by the calculated the comparison made with that of the results obtained by SPICE simulations.

\section{REFERENCES}

[1] Elmore, W.C., "The transient response of damped Linear network with Particular regard to Wideband Amplifiers", J. Applied Physics, 19, 1948, pp.55-63.

[2] Kay, R., Pileggi, L. PRIMO: Probability Interpretation of Moments for Delay Calculation, IEEWACM Design Automation Conference, 1998, pp. 463-468.

[3] Yang Wu, Shien, Boon-Khim Liew, K.L. Young, C.H.Yu, and S.C. Analysis of Interconnect Delay for $0.18 \mu \mathrm{m}$ Technology and Beyond" IEEE International Conference Interconnect Technology, May 1999 , pp. 68 - 70

[4] Lin, T. , Acar, E. , Pileggi, L., h-gamma: An RC Delay Metric Based on a Gamma Distribution Approximation to the Homogeneous Response, IEEE/ACM International Conference on Computer-Aided Design, 1998, pp. 19-25.

[5] Pillage, L. T., Rohrer, R. A., Asymptotic Waveform Evaluation for Timing Analysis, Tran. on CAD, Volume 9, Issue 4, 1990. pp. 331- 349

[6] Gupta, R., Tutuianu, B., Pileggi, L. T. , The Elmore Delay as a Bound for RC Trees with Generalized Input Signals, IEEE Trans. on CAD, 16(1), pp. 95-104, 1997.

[7] Larson, H. J., Introduction to Probability Theory and Statistical Inference, 3rd ed., John Wiley \& Sons pub., 1982

[8] Kendall M. G., Stuart, A., The Advanced Theory of Statistics, vol. 1: Distribution Theory, New York: Hafner, 1969.

[9] MacGillivray, H. L., The Mean, Median, Mode Inequality and Skewness for a Class of Densities, Australian J. of Statistics, vol. 23 no. 2, 1981

[10] Chu C., Horowitz, M., Charge-Sharing Models for Switch Level Simulation, Trans. on CAD, Volume 6, Issue 6 June, 1987.

[11] Harald Cramer. Mathematical Methods of Statistics. Princeton University Press, 1946.

[12] Celik, L. Pileggi, Odabasioglu, A., IC Interconnect Analysis, Kluwer Academic Publishers, 2002. 